\title{
THREE-DIMENSIONAL RECONSTRUCTION OF THE VIRTUAL PLANT BRANCHING STRUCTURE BASED ON TERRESTRIAL LIDAR TECHNOLOGIES AND L-SYSTEM
}

\author{
Yinxi Gong ${ }^{1,2}$, Yanqin Yang ${ }^{1,2}$, Xiaofen Yang ${ }^{1,2}$ \\ 1. National Administration of Surveying, Mapping and Geoinformation engineering research center of Geographic National \\ Conditions Monitoring, Xian 710000, China; \\ ${ }^{2}$ The First Institute of Photogrammetry and Remote Sensing, Xian 710000, China;
}

KEY WORDS: Terrestrial LiDAR, L-system, Branching structure, Point cloud data, Topology structure

\begin{abstract}
:
For the purpose of extracting productions of some specific branching plants effectively and realizing its 3D reconstruction, Terrestrial LiDAR data was used as extraction source of production, and a 3D reconstruction method based on Terrestrial LiDAR technologies combined with the L-system was proposed in this article. The topology structure of the plant architectures was extracted using the point cloud data of the target plant with space level segmentation mechanism. Subsequently, L-system productions were obtained and the structural parameters and production rules of branches, which fit the given plant, was generated. A threedimensional simulation model of target plant was established combined with computer visualization algorithm finally. The results suggest that the method can effectively extract a given branching plant topology and describes its production, realizing the extraction of topology structure by the computer algorithm for given branching plant and also simplifying the extraction of branching plant productions which would be complex and time-consuming by L-system. It improves the degree of automation in the L-system extraction of productions of specific branching plants, providing a new way for the extraction of branching plant production rules.
\end{abstract}

\section{INTRODUCTION}

As the structure of branching plants closely related to the process of their growth and development, accurately and quickly simulating three-dimensional (3D) structure of the branching plants can effectively and precisely show the process of plant growth and development, and reflect the growth law. In recent years, as the increasing cross fusion of life science and information science, it is possible to digitize the structures and functions of plants and thus achieve a precise description, visual expression, development evaluation, quantitative calculation, simulation and prediction of the plant morphology. And it has become an important and developing direction of the contemporary agricultural science and forestry production (Prusinkiewicz, 2004). Virtual plant has been taken as a useful tool to understand complex relationship among plant gene function, plant growth, plant physiology and plant form.

A series of Worldwide research has been conducted on the structure model of virtual plants, and significant results have been achieved (Zhao, De Reffye, Xiong Hu, Zhan, 2001). Primarily, they are the Lindenmayer system (L-system) put forward by American biologist Lindenmayer in 1968 (Lindenmayer, 1968), "particle system" put forward by Reeves (Reeves, 1983) and iterative function system(IFS) proposed by M.F.Barnsley and also the Modeling method through reference axis technology proposed by De Reffye (De Reffye, Edelin, Franqon, Jaeger, Puech, 1988), CIRAD. But plant models generated by the IFS and other graphics methods that less consider botany knowledge are difficult to couple the plant physiological and ecology models, which causes small diversity in the morpha of simulating Plants (Godin,Caraglio, 1998). Particle system is a modeling method put forward to "fuzzy" objects. It is suitable for simulating forest, grass and other macro- objects, but lack of reality sense for the simulation of branching structures of a single plant(Magdics, 2009). And Lsystem can combine the botanical theory to capture plant topology structures and easily convert them into the language recognized by computers, which can dynamically simulate growing process of trees. So it is very suitable for describing the morphological structure of a single plant. In order to establish the plant models more effectively, the functions of the L-system were constantly improved. D0L- system, proposed by Prusinkiewicz (Prusinkiewicz, Rolland-Lagan, 2006), using turtle graphics and combining random L-system, can construct random topology structure of the plant. Parametric L-system formed one that could simulate the plant growth process better (McCormack, 2005). Honda's application of the certain algorithm defined the branch structures of plants. By changing parameters of the length of the branches, shrinkage and branch angles, all sorts of tree models and more realistic simulation results were obtained (Honda, Tomlinson, Fisher, 1982). Other scholars also improved L-system and put forward the random Lsystem, extended L-system (Kniemeyer, 2004), open L-systems (Měch, Prusinkiewicz, 1996). Soler and others, basing on improved L-system achieved modeling (Soler, Sillion, Blaise, Dereffye, 2000). While Zhao Xing raised the dual-scale automata (Zhao, De Reffye, Xiong Hu, Zhan, 2001) and so on. Improvements of these studies on L-system are all in order to improve simulation performance of the L-system, to improve the simulation of plant continuous growth process, and adding the impact of plant-environment interactions may aim to enrich the species of the simulation plants.

However, because L-system uses productions to modeling, its productions are generally described in accordance with the plant growth process (Prusinkiewicz, 1998). Previous extraction of productions was achieved by manual analyzing the morphology structure of the target plants, using the method of aritificial statistical and manual measurement, and productions of the Lsystem were extracted by statistical data. In most cases it was needed to manually set the operating parameters by experience and estimation, but the description of the process was not intuitional and also there were more uncertainties. For woody branching plants which are more complex in the distribution of spatial structure, the descriptions were of low efficiency or even difficult to achieve (Bedrich Benes, Erik Uriel Millian, 2002). As for 3D reconstruction of branching structure of given plants, previous L-systems often used two-dimensional images as data 
sources of branching plants, and often required artificial recognition in extracting the branching structure. The consistency of the results with the prototype plants is less than ideal (Bian, Chen, Burrage, Hanan, Room, Belward, 2002). The extracted productions were also difficult to understand for nonprofessional program staff, so the applicability of L-system in the $3 \mathrm{D}$ reconstruction of the branching structures of given plants was affected.

Considering the defects above, in this paper we put forward a new 3D reconstruction method for the branching structure of the given plants, based on the Terrestrial LiDAR data combined with L-system. The Terrestrial LiDAR is a kind of increasingly mature technology and is widely used in the reverse engineering field. As a kind of technique with which we can obtain space structure of target objects accurately and fast, it can be used to realize fast reconstruction of the spatial structures of target objects with high accuracy (Garber, Monserud, Mguire, 2008), and then extract the structural parameters of targets. In this paper, combining the L-system and cloud point data of the Terrestrial LiDAR from which 3D information of plant space can be obtained, and with the characteristics of the projection of branch point clouds approximating the arc structure, parameters and the topology structures of the branches of target plants can be extracted. And using structures of quasi binary trees to simplify the production of branching plants, the 3D reconstruction can be achieved. Experimental results show that using this method plant topology structures can effectively be extracted and also the plant production rules can be described, overcoming the previous disadvantages of excessive reliance on empirical models, improving the operability of extracting the productions of branching plants and providing a new way for the extraction of plant productions.

\section{MATERIALS AND METHODS}

\subsection{Terrestrial LiDAR point cloud data acquisition}

Terrestrial 3D LiDAR system was used in this study as data source. Experimental instrument was FARO Focus 3D laser scanner. Vertical scanning Angle was $305^{\circ}$ and horizontal scanning angle was $360^{\circ}$. The highest resolution can be up to $0.9 \mathrm{~mm}$ of point spacing at a distance of $10 \mathrm{~m}$. The instrument is characterized by a wide range of the vertical angle, high emission frequency, and it can fully scan the information of plant branches and improve the efficiency of splicing of different sites. By using a synchronization digital camera Nikon D700 with high-resolution and a wide angle, and then the high resolution photos superposed with the $3 \mathrm{D}$ point cloud information made the extraction of target information greatly facilitated. When scanning, matching balls were evenly placed at the sample plot, and the matching balls were used to splice the point cloud. Thus the accuracy and speed of the registration were greatly improved (Omasa, Hosoi, Konishi, 2007). In order to get the omnibearing information of point cloud of the prototype plants, at least three stations of the scanning are needed on the same target to get the complete spatial information of plants (Parker, Harding, Berger, 2004). The principles of scanning the sample plot should be: to get the information of the spatial position of all the trees in the sample plot with the least number of stations. For if the stations are too many, extra work will be added to the splicing of the point cloud data. This problem can be eliminated by optimizing the arrangement of control points in the process of collecting data. As shown in figure 1, the following was the basic principle in the control point layout in the data acquisition. By screening, in this study a Locust and an Oak whose scan data are the best in splicing effects were chosen to be the prototype plants of the $3 \mathrm{D}$ reconstruction.

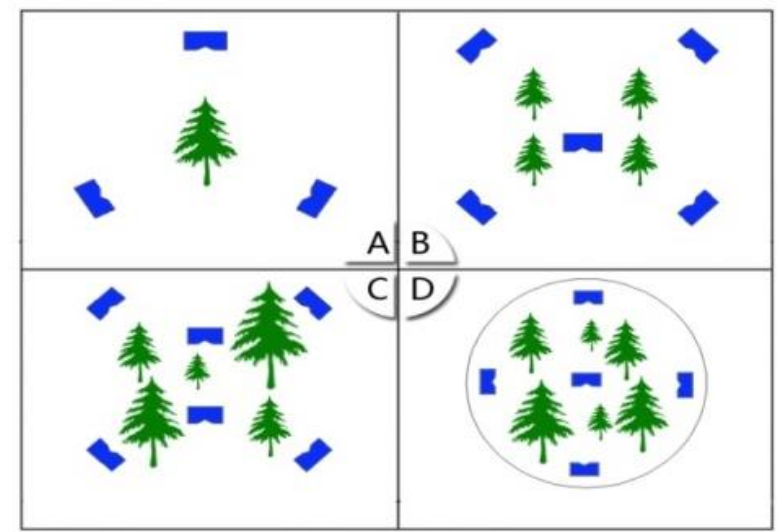

Figure 1. The principle of control point arrangement

\subsection{D L-system}

L-system is an important part of the fractal theory, and its core concept is to rewrite. The method of rewrite is achieved through the application of a collection of rewrite rules, and it is a technology of defining the complex targets by continuously replacing parts of the simple initial targets (Karwowski, Prusinkiewicz, 2003).

3D L-system was achieved by popularizing geometric explanation of the turtle graphics in the L-system. Its key point is that the current position of the turtle graphics in space is represented by the three vector $[\mathrm{H}, \mathrm{L}, \mathrm{U}]$, where $\mathrm{H}$ represents forward, L left and U up. And direction vectors are mutually perpendicular unit vectors, that is, $\mathrm{H} \times \mathrm{L}=\mathrm{U}$. Rotation of the turtles can be represented by $[\mathrm{H}, \mathrm{L}, \mathrm{U}]=[\mathrm{H}, \mathrm{L}, \mathrm{U}] \cdot \mathrm{R}$ (Prusinkiewicz, 2004). Among them:

$$
\begin{aligned}
& \mathrm{R}_{\mathrm{H}}(\alpha)=\left[\begin{array}{ccc}
1 & 0 & 0 \\
0 & \cos \alpha & -\sin \alpha \\
0 & \sin \alpha & \cos \alpha
\end{array}\right] \\
& \mathrm{R}_{\mathrm{H}}(\alpha)=\left[\begin{array}{ccc}
1 & 0 & 0 \\
0 & \cos \alpha & -\sin \alpha \\
0 & \sin \alpha & \cos \alpha
\end{array}\right] \\
& \mathrm{R}_{\mathrm{v}}(\alpha)=\left[\begin{array}{ccc}
\cos \alpha & \sin \alpha & 0 \\
-\sin \alpha & \cos \alpha & 0 \\
0 & 0 & 1
\end{array}\right]
\end{aligned}
$$

Its spatial rotation diagram shown in Figure 2 


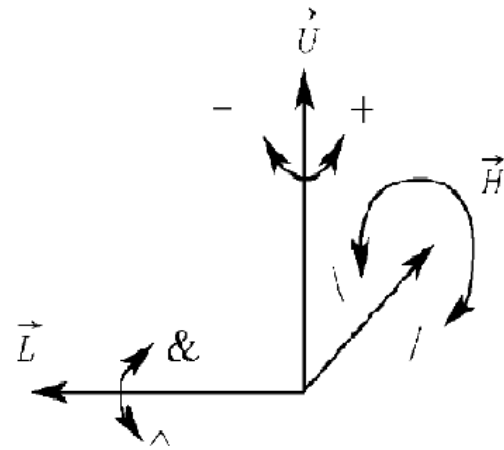

Figure 2. Space rotation diagram of 3D L-system

Legends: $\mathrm{H}$ is forward, $\mathrm{L}$ for left and $\mathrm{U}$ for up, and the direction vector is mutually perpendicular unit vector.

\subsection{Quasi binary tree}

In this paper the definition of a quasi binary tree is the extension of binary tree. It consists of binary trees and other multiple trees (Gary D. Knott, 1997). The following are the related properties of binomial trees: For the complete binary tree with $\mathrm{N}$ nodes $\mathrm{Y}$, set $\mathrm{j}(1 \leq \mathrm{j} \leq \mathrm{n})$ is the $\mathrm{j}$-th node of the order storage sequence, so when $\mathrm{j}=1$, the node $\mathrm{j}$ is the root node with no parents. Otherwise, the parents of $\mathrm{j}$ should be $\lfloor\mathrm{j} / 2\rfloor$, that is, $\lfloor j / 2\rfloor$ as root, two nodes $j$ and $j+1$ are separated out.

If $\mathrm{Y}$ is a full binary tree with depth $\mathrm{n}$, it has $2^{n}-1$ nodes. The subtree whose root is an arbitrary node of the layer $\mathrm{K}(0<\mathrm{k} \leq \mathrm{n})$ has $2^{n-k+1}-1$ nodes. Similarly, according to the properties of binary trees, the related properties of other bifurcate tree structure can be derived.

\subsection{D reconstruction strategy}

According to the fractal theory, fractal objects described with fractal methods should have the following characteristics: Firstly, described object has self-similarity between its parts and whole. Secondly, from self-similarity of the fractal objects the appropriate similar structure can be extracted to carry out the simulation of fractal objects (Fang, Jing, Shen, Chen, 2011). As can be seen from Figure 3, a 3D model of a branching plant is a combination of nodes and branches with topological relations in 3D space, and this combination, after being simplified, can render a 3D structure of a quasi binary tree. That is, the relation between the nodes of branching plant stems and branches of the branching plant is of related properties of a quasi binary tree. In the data structure, a binary tree is a two dimensional structure. When it is needed to use two dimensional structure to build a 3D model, we can obtain the spatial structure model of quasi binary trees just by rotating the quasi binary tree in space. This quasi binary tree model with 3D structure has the same branch topology structure with the prototype plant. Through recognition of the topology structure, the base of the simulation parameters will be provided for L-system.

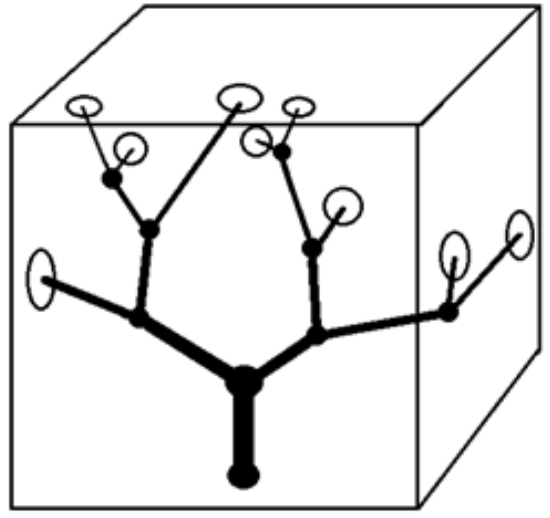

Figure 3. 3D structure of a plant

In the parameter extraction of branching plant structures, Todd $\mathrm{k}$ and Sinoquet $\mathrm{H}$ raised two different methods of branch recognition (Todd, Csillag, Atkinson, 2003. Sinoquet, Sonohat, Phattaralerphong, Godin, 2005). The premise of these two methods application is that the point cloud of branches distributes in a arc shape in the horizontal direction. For smaller plants and the plants which are far from the scanner, if the sampling frequency is lower, it is hard to ensure to meet the conditions. Unlike these methods, in this paper, we took a single tree for example, and got its point cloud data from multiple angles and close distance. Identification algorithm on the spatial structure of a branching tree was carried out by using the characteristics that point cloud of branches continuously distribute in their growth direction, and in the process, the parameters of both the bifurcation nodes and spatial location of the branches could be obtained from the point cloud information of the target trees. The extraction schematic diagram of the spatial location and the radius of branches is shown as in Figure 4.

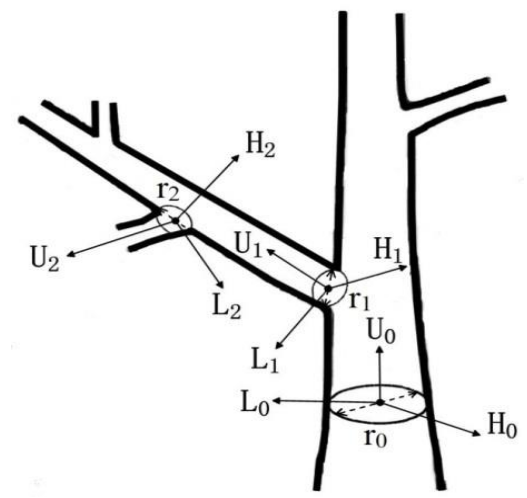

Figure 4. Branch parameter extraction

Legends: $\left[\mathrm{H}_{\mathrm{i}}, \mathrm{L}_{\mathrm{i}}, \mathrm{U}_{\mathrm{i}}\right]$ is $3 \mathrm{D}$ vectors stand for the growing direction of branches at different nodes, $\mathrm{r}_{i}$ is the radius of the branches at different nodes.

Therefore, the modeling process of branching plants mainly consists of three steps:

a) Using $F_{i j}$ to represent the simplest branch, first by analyzing the point cloud data of target plants, extract the topology information of branching plants and parameters information for each layer of distinguishable branches $F_{i j}(d, 1, r, a)$, of which $i$ is the number of layers, $j$ is the serial number and " $d$ ", the growth direction of branches, comes from a $3 \mathrm{D}$ vector $\left[\mathrm{H}_{\mathrm{i}}, \mathrm{L}_{\mathrm{i}}, \mathrm{U}_{\mathrm{i}}\right]$; "l" represents the length of branches, worked out from the Euclidean norm from the 3D vector $\mathrm{F}_{\mathrm{ij}}(\mathrm{d})$ of adjacent branch 
node and $\mathrm{F}_{\mathrm{i}+1 \mathrm{j}}(\mathrm{d})$, its formula is as follows: $1=\overline{\left\|\mathrm{F}_{i, j} \mathrm{~F}_{i+1, j}\right\|}$; " $\mathrm{r}$ " is the radius of the drawn branches, extracting from the formula $\mathrm{r}=C / 2 \pi$, in which $\mathrm{C}$ is the perimeter of the contours of the point cloud cross-section fitting; "a" represents the attenuation coefficient of the branches, resulted from the formula $\mathrm{F}_{\mathrm{ij}}(\mathrm{a})=\mathrm{F}_{i+1, j}(\mathrm{r}) / \mathrm{F}_{i, j}(\mathrm{r})$, in which $\mathrm{F}_{\mathrm{ij}}(\mathrm{r})$ is the branch radius at the node of the adjacent branches.

b) Then through generalizing and abstracting the structures combined with the characteristic parameters of branching plants, form the axiom (the starting strings) and production rules, and further iteratively generate a character sequence representing tree structures.

c) Finally translate each character in the L-system into corresponding graphic elements, and combining with the already extracted production rules as well as using 3D L-system, achieve the goal of 3D reconstruction of target plants.

\subsection{Extraction algorithm of branching plant topology structure based on point cloud data}

From the view of biology, growing and developing of plants is a natural process of continuous changes (Godin, Sinoquet, 2005). So we can think the spatial location of the barycenter of the same structure in the continuous cross-section is of continuous change. That is to say, their trajectories in space are a set of line segments of geometric continuity.

On the basis of the theory above, according to the spatial structures of branching plant, in this article we propose a topology extraction algorithm of branching plant based on barycenter. In the algorithm, space is firstly divided into hierarchical grids, and the point cloud on each grid is projected onto a two-dimensional plane. Using fitting method, the discrete point cloud data is fitted into two-dimensional graphics with polygon boundary; Then, through computer graphic analysis, the cross-section of branching plant is formed; Finally, by detecting the continuous distribution of barycenter of faults in vertical direction, the topology structures of the branching plant are reconstructed. Thus, we can accurately determine the spatial locations of plant stems, geometric shapes and the spatial relationships between the stems, and thus we get the topology structures of branching plant. The following are the topology structure of branching plant of this case, as shown in Figure 5, which corresponds to the adjacent lists of node to node, branch to branch, shown in table 1 , table 2 .

\begin{tabular}{|c|c|c|c|c|}
\hline Node & Parent & Child & $\begin{array}{c}\text { Coordinate (x, } \mathrm{y}, \\
\mathrm{z})\end{array}$ & $\begin{array}{l}\text { Radius } \\
\text { (r) }\end{array}$ \\
\hline $\mathrm{N}_{11}$ & - & $\mathrm{N}_{21}$ & $\mathrm{~N}_{11}(\mathrm{x}, \mathrm{y}, \mathrm{z})$ & $\mathrm{N}_{11}(\mathrm{r})$ \\
\hline $\mathrm{N}_{21}$ & $\mathrm{~N}_{11}$ & $\begin{array}{c}\mathrm{N}_{31}, \mathrm{~N}_{32}, \\
\mathrm{~N}_{33}\end{array}$ & $\mathrm{~N}_{21}(\mathrm{x}, \mathrm{y}, \mathrm{z})$ & $\mathrm{N}_{21}(\mathrm{r})$ \\
\hline $\mathrm{N}_{31}$ & $\mathrm{~N}_{21}$ & $\mathrm{~N}_{41}$ & $\mathrm{~N}_{31}(\mathrm{x}, \mathrm{y}, \mathrm{z})$ & $\mathrm{N}_{31}(\mathrm{r})$ \\
\hline $\mathrm{N}_{32}$ & $\mathrm{~N}_{21}$ & - & $\mathrm{N}_{32}(\mathrm{x}, \mathrm{y}, \mathrm{z})$ & $\mathrm{N}_{32}(\mathrm{r})$ \\
\hline $\mathrm{N}_{33}$ & $\mathrm{~N}_{21}$ & $\mathrm{~N}_{42}, \mathrm{~N}_{43}$ & $\mathrm{~N}_{33}(\mathrm{x}, \mathrm{y}, \mathrm{z})$ & $\mathrm{N}_{33}(\mathrm{r})$ \\
\hline $\mathrm{N}_{41}$ & $\mathrm{~N}_{31}$ & $\begin{array}{c}\mathrm{N}_{51}, \mathrm{~N}_{52}, \\
\mathrm{~N}_{53}\end{array}$ & $\mathrm{~N}_{41}(\mathrm{x}, \mathrm{y}, \mathrm{z})$ & $\mathrm{N}_{41}(\mathrm{r})$ \\
\hline $\mathrm{N}_{42}$ & $\mathrm{~N}_{33}$ & - & $\mathrm{N}_{42}(\mathrm{x}, \mathrm{y}, \mathrm{z})$ & $\mathrm{N}_{42}(\mathrm{r})$ \\
\hline $\mathrm{N}_{43}$ & $\mathrm{~N}_{33}$ & $\mathrm{~N}_{54}$ & $\mathrm{~N}_{43}(\mathrm{x}, \mathrm{y}, \mathrm{z})$ & $\mathrm{N}_{43}(\mathrm{r})$ \\
\hline $\mathrm{N}_{51}$ & $\mathrm{~N}_{41}$ & - & $\mathrm{N}_{51}(\mathrm{x}, \mathrm{y}, \mathrm{z})$ & $\mathrm{N}_{51}(\mathrm{r})$ \\
\hline $\mathrm{N}_{52}$ & $\mathrm{~N}_{41}$ & - & $\mathrm{N}_{52}(\mathrm{x}, \mathrm{y}, \mathrm{z})$ & $\mathrm{N}_{52}(\mathrm{r})$ \\
\hline $\mathrm{N}_{53}$ & $\mathrm{~N}_{41}$ & - & $\mathrm{N}_{53}(\mathrm{x}, \mathrm{y}, \mathrm{z})$ & $\mathrm{N}_{53}(\mathrm{r})$ \\
\hline $\mathrm{N}_{54}$ & $\mathrm{~N}_{43}$ & - & $\mathrm{N}_{54}(\mathrm{x}, \mathrm{y}, \mathrm{z})$ & $\mathrm{N}_{54}(\mathrm{r})$ \\
\hline
\end{tabular}

Table 1. Adjacency lists of the node-node topology relationship Note: Where $\mathrm{N}_{\mathrm{ij}}(\mathrm{x}, \mathrm{y}, \mathrm{z})$ is the node coordinates, $\mathrm{N}_{\mathrm{ij}}(\mathrm{r})$ is branch radius at the nodes

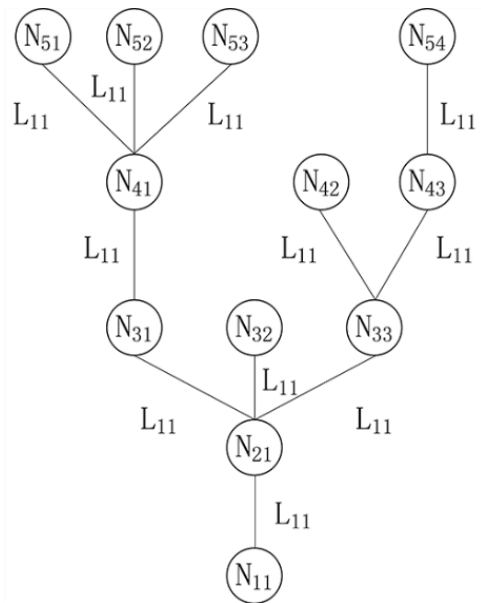

Figure 5. Topology example of branch segments - node

Legends: $\mathrm{N}_{\mathrm{ij}}$ is nodes, $\mathrm{L}_{\mathrm{ij}}$ is branch segments.

\begin{tabular}{|c|c|c|}
\hline Branch & Parent & Child \\
\hline $\mathrm{L}_{11}$ & - & $\mathrm{L}_{21}, \mathrm{~L}_{22}, \mathrm{~L}_{23}$ \\
$\mathrm{~L}_{21}$ & $\mathrm{~L}_{11}$ & $\mathrm{~L}_{31}$ \\
$\mathrm{~L}_{22}$ & $\mathrm{~L}_{11}$ & - \\
$\mathrm{L}_{23}$ & $\mathrm{~L}_{11}$ & $\mathrm{~L}_{32}, \mathrm{~L}_{33}$ \\
$\mathrm{~L}_{31}$ & $\mathrm{~L}_{21}$ & $\mathrm{~L}_{41}, \mathrm{~L}_{42}, \mathrm{~L}_{43}$ \\
$\mathrm{~L}_{32}$ & $\mathrm{~L}_{23}$ & - \\
$\mathrm{L}_{33}$ & $\mathrm{~L}_{23}$ & $\mathrm{~L}_{44}$ \\
$\mathrm{~L}_{41}$ & $\mathrm{~L}_{31}$ & - \\
$\mathrm{L}_{42}$ & $\mathrm{~L}_{31}$ & - \\
$\mathrm{L}_{43}$ & $\mathrm{~L}_{31}$ & - \\
$\mathrm{L}_{44}$ & $\mathrm{~L}_{33}$ & - \\
\hline
\end{tabular}

Table 2. Adjacency lists of the segment-segment topology relationship of branches

Note: Where $\mathrm{L}_{\mathrm{ij}}$ is branch segments.

Extended with the node $\mathrm{N}_{11}$ as the starting point, the resulting adjacent lists of topological relationship of the branch segmentnode are shown in Table 3.

\begin{tabular}{|c|c|c|c|}
\hline Branch & Start point & End point & Length \\
\hline $\mathrm{L}_{11}$ & $\mathrm{~N}_{11}$ & $\mathrm{~N}_{21}$ & $\mathrm{~L}_{11}(1)$ \\
$\mathrm{L}_{21}$ & $\mathrm{~N}_{21}$ & $\mathrm{~N}_{31}$ & $\mathrm{~L}_{21}(1)$ \\
$\mathrm{L}_{22}$ & $\mathrm{~N}_{21}$ & $\mathrm{~N}_{32}$ & $\mathrm{~L}_{22}(1)$ \\
$\mathrm{L}_{23}$ & $\mathrm{~N}_{11}$ & $\mathrm{~N}_{33}$ & $\mathrm{~L}_{23}(1)$ \\
$\mathrm{L}_{31}$ & $\mathrm{~N}_{21}$ & $\mathrm{~N}_{41}$ & $\mathrm{~L}_{31}(1)$ \\
$\mathrm{L}_{32}$ & $\mathrm{~N}_{23}$ & $\mathrm{~N}_{42}$ & $\mathrm{~L}_{32}(1)$ \\
$\mathrm{L}_{33}$ & $\mathrm{~N}_{23}$ & $\mathrm{~N}_{43}$ & $\mathrm{~L}_{33}(1)$ \\
$\mathrm{L}_{41}$ & $\mathrm{~N}_{31}$ & $\mathrm{~N}_{51}$ & $\mathrm{~L}_{41}(1)$ \\
$\mathrm{L}_{42}$ & $\mathrm{~N}_{31}$ & $\mathrm{~N}_{52}$ & $\mathrm{~L}_{42}(1)$ \\
$\mathrm{L}_{43}$ & $\mathrm{~N}_{31}$ & $\mathrm{~N}_{53}$ & $\mathrm{~L}_{43}(1)$ \\
$\mathrm{L}_{44}$ & $\mathrm{~N}_{33}$ & $\mathrm{~N}_{54}$ & $\mathrm{~L}_{44}(1)$ \\
\hline
\end{tabular}

Table 3. Adjacency lists of the segment-node topological relation

From the topology relations above, the parameter information of each segment of branches can be obtained. Take extracting the parameters of the segment $F_{11}(d, 1, r, a)$ as an example, the branch segment belonging to $F_{11}$ is $L_{11}$, so $F_{11}$ gets the topology information of $\mathrm{L}_{11}$. Therefore the starting point coordinate of $\mathrm{F}_{11}$ (d) is $\mathrm{N}_{11}(\mathrm{x}, \mathrm{y}, \mathrm{z})$, the end point coordinate is $\mathrm{N}_{21}(\mathrm{x}, \mathrm{y}, \mathrm{z})$ and the $3 \mathrm{D}$ vector $\left[\mathrm{H}_{11}, \mathrm{~L}_{11}, \mathrm{U}_{11}\right]$ can be obtained by the two 
coordinates; $F_{11}$ (l) is equal to $\mathrm{L}_{11}(1) ; \mathrm{F}_{11}(\mathrm{r})$ is equal to $\mathrm{N}_{11}(\mathrm{r})$; and $F_{11}$ (a) can be resulted from the ratio of $F_{21}(r) / F_{11}(r)$. Thus all the parameter information in the $\mathrm{F}_{11}(\mathrm{~d}, 1, \mathrm{r}$, a) can be obtained.

The following is the specific extraction process of the algorithm: Step1: Read the point cloud data of target plants, namely the trees needing 3D reconstruction.

Step2: Extract the point clouds of fault trees at certain intervals and thicknesses (the interval and thickness are based on the complexity of the trees), and the numbers are adjacent between the faults.

Step3: Project the point clouds of each fault to a plane vertically and respectively, and then use the quadratic Laplacian to exclude discrete noise points (Felsberg, Sommer, 2001), excluding the interference of leaves and small branches, and making the stems and main branches the remaining parts; Step4: Form one or more two-dimensional graphics with closed polygons by fitting the projection graphics in Step3, and extract the contours of branchs. As shown in figure 6 a shows;

Step5: use P.Guldin theorem (Yang, 2004) to work out the barycenters for all the closed two-dimensional graphics of each fault, extract the barycenters of branches and stems. As shown in figure $6 \mathrm{~b}$.

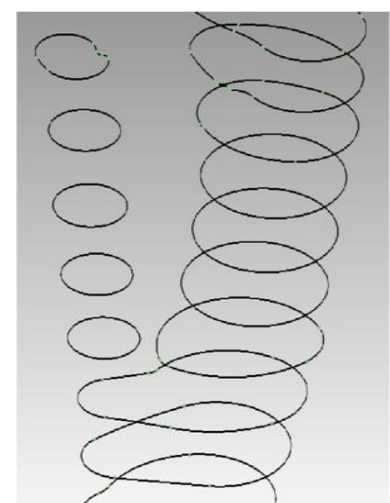

a

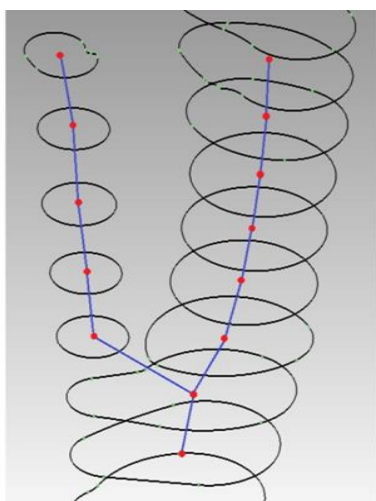

b
Figure 6. Branch contour fitting and extraction of barycenters Legends: The red points represent the barycenter, and blue segments are the growth direction of branches.

Step6: After connecting the barycenters of branches between each adjacent layer through line segments, we actually get the spatial locations of nodes on each branch layer, radius $r_{i}$ of each branch segment and also the spatial location of each branch represented by the three vectors $\left[\mathrm{H}_{\mathrm{i}}, \mathrm{L}_{\mathrm{i}}, \mathrm{U}_{\mathrm{i}}\right]$ in the $3 \mathrm{D} \mathrm{L}$-system, and accordingly get a topology structure of the prototype tree branches, as shown in Figure 7;

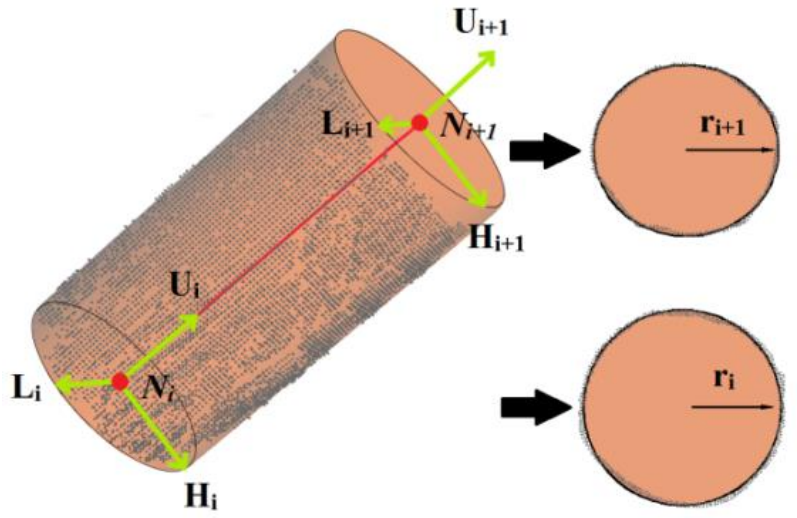

Figure 7. Extraction of the spatial location and radius of branches

Legends: $r_{i}$ is each branch segment radius, $\left[\mathrm{H}_{i}, \mathrm{~L}_{\mathrm{i}}, \mathrm{U}_{\mathrm{i}}\right]$ is three vectors of each branch direction, $\mathrm{N}_{\mathrm{i}}$ is number of each adjacent layer.

Step7: Following the topology of tree branches, extract the type of branches, make adjustments to the structure models of branches, integrate the branching structures of various types, and build the corresponding structure models of quasi binary trees. In this article we define $\mathrm{Y}$ is the binary branch, and $\mathrm{K}$ the three forks branch;

Step8: Simplify the topology models of tree branches, until the most simple branches, shown in Figure 8;

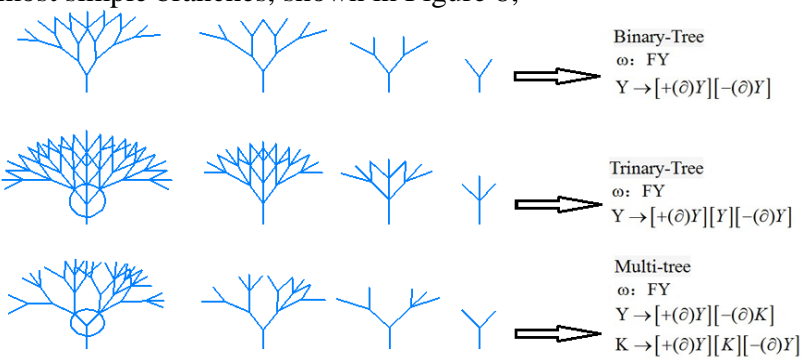

Figure 8. Simplification process and iterative rules of branches structure

Step9: Extract the iteration number $\mathrm{n}$ and the type and the parameters of each branch according to the topology model of prototype trees in Step6, and establish character tables of the simplest branches of various types, including the length 1 of each simplest branches, radius $\mathrm{r}$ of branches, and other key elements as growth direction $\mathrm{d}$;

Step10: Determine the basic iteration rules of Y, K and axioms $\omega$ of all kinds of branches;

Step11: According to the character table of the most simple branches, perfect the basic iteration rules, establish a complete production, and thereby establish a complete L-system model.

\subsection{Branch reconstruction based on $L$-system}

On the proposed method above, some related indexes can be obtained: branch type $\mathrm{Bt}$, physiological cycle (called the iteration number) $\mathrm{n}$ of plant growth and the morphological structure of plants, and production for extraction L-system modeling. And according to the nature of quasi binary trees of branching plants, branching number $n u m \leq 2^{(n-1)}-1$, node 
number $\operatorname{Nod}_{n}=\sum_{\mathrm{i}=1}^{n} m_{i} \times \mathrm{i}$, in which $1 \leq \mathrm{n} \leq \mathrm{bt}, \quad$ mun $_{i}$ is the number of different branches, namely the product of each different branching type and the branch. Therefore, the Lsystem based on the method in this paper can be defined as:

1) $\omega$ : the minimalist branches

2) Iteration number $n$ : the layer number $n$ of the topology structure of plants

3) Production p: first, determine the basic production of each branching type, according to the number Bt of branch types, such as binary branches $[\mathrm{Y}][\mathrm{Y}]$, three forks branch $[\mathrm{K}][\mathrm{K}][\mathrm{K}]$ and then according to the 3D coordinates of the nodes of each layer in the topology structure of plants, said with 3D L-system vectors $[\mathrm{H}, \mathrm{L}, \mathrm{U}]$, and combining with the most simple branches of the layers which contain the information of branch length "l", branch radius " $r$ " as well as the attenuation coefficient "a" of the branches, thus establish reasonable production which is consistent with the prototype trees.

By the process of the production description, the structure and number of production $\mathrm{p}$ is decided by the branching structure and spatial location of nodes of each layer. Their numbers can be expressed as:

$$
\mathrm{Bt} \leq \mathrm{p}_{\mathrm{i}}<2^{n-1}-1 \quad\left(\mathrm{i} \in \mathrm{R}^{*}\right)
$$

That is, the more branch types Bt and the branch number, the more the production $\mathrm{P}_{\mathrm{i}}$ the greater the total number of growth units $\mathrm{S}$, and the more complex the generated $3 \mathrm{D}$ structures of plants.

Because the production description of L-system is closely related with the growing process of plants (Pradal, DufourKowalski, Boudon, Fournier, Godin, 2008), and both the production number and the structure description are of uncertainty (Federl, Prusinkiewicz, 2004), the following relation exists between the number of productions and branch types Bt:

$$
\mathrm{Bt} \leq \mathrm{p}_{\mathrm{i}}<\infty\left(\mathrm{i} \in \mathrm{R}^{*}\right)
$$

From the comparison of (1), (2) and the describing process of production $\mathrm{p}$ in this paper, in production number and the description for each rule, the method in this article has strong operability with certainty.

\section{RESULTS AND DISCUSSION}

Using C++ in the Visual Studio 2008, in this article we have studied and developed the simulation system for 3D structure reconstruction of branching plants by Terrestrial LiDAR data. The defined data structure in the research and the codes of the related main program are as follows:

Typedef struct TreeNode

\section{\{}

unsigned Index;

char Axiom; // Axiom

int Layers; // Iteration times

char Rule[num]; // Production ruls

char Bt[n]; // Branch types

float Direction[n]; // The direction vectors of branches in space \} void CTreeView::Draw()

\{

InitDatas(); // Initialized data

void $(* \mathrm{p})()$; // Define pointer variables to a function

$\mathrm{p}=\mathrm{E}$; //The entry address of a function of recording information passing

ConstructTree(); // Construct plant production

DrawTree() ; // Reconstruct 3D shapes of plants according to the productions

\}

In the process of reconstruction, first analyze the detached point cloud information of prototype tree, and extract the parameter information of topology structures of the tree and identifiable branches. And then by generalization and abstraction of the topology and parameters and building production rules, progressively and iteratively generates a character sequence of the tree structure. Finally, each character in the L-system is interpreted into corresponding graphic elements, and using the 3D L-system, 3D reconstruction of trees is realized. The simulation results are shown in Figure 9 and Figure 10.

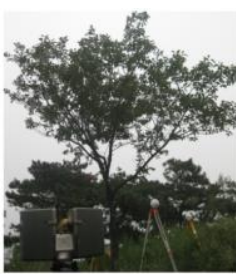

a)

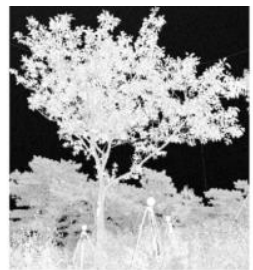

b)

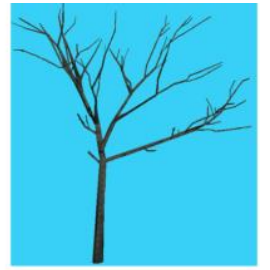

Figure 9. Simulation results of an Oak

Legends: a) Scene photographs of the Oak prototype. b) Point cloud screenshot of the Oak prototype. c) Simulation result

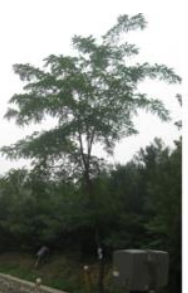

a)

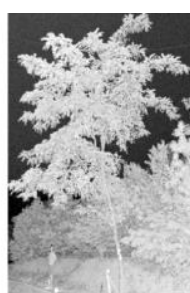

b)

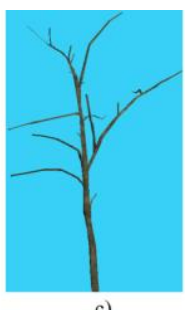

Figure 10. Simulation results of a Locust

Legends: a) Scene photo of the Locust prototype. b) Point cloud Screenshot of the Locust prototype. c) Simulation results

In Fig. 9 and 10, the results of the 3D reconstruction of trees show that the models of trees constructed by the method proposed in this article are highly consistent with the prototype trees. Most branches have achieved reasonable reconstruction effects, and expressions of the main branches are correct. The 3D reconstruction of the branching plants has been achieved. But it can also be seen that the simulation results still need to be improved. Taking c) in figure 10 for example, we can clearly see that not all branches have been reconstructed, and that some smaller branches have not been presented or the expressions are incomplete. This is due to the reasons that the dense leaves shade, that the branches are too small, and also that the branch growth is more irregular, causing the branches to be filtered out by denoising algorithm or fail to pass the algorithm identification. For this kind of problems, we are considering to resolve by means of improving the topology extraction algorithm or manual intervention in future studies, but the manual intervention will reduce automation degree the method 
proposed in this paper.

And it can also be found from c) in fig. 9 and c) in fig. 10 that branches of the trees did not show the natural shape of curve. This is because the geometry models of branches in the parser uses a round platform, and so it is difficult to simulate the branches with curvature. In the further study generalized cylinder will be used in the geometric expression of branches, and thus the branches in the form of curve can be simulated. Due to accidental errors of the field data, parts of the point cloud data of the target plants require artificial corrective treatment, with which the 3D reconstruction of trees can be of better results. In this paper, in the future research we will consider the introduction of the method of smooth fitting of the point cloud, which will make the method in this article more automating.

Sample data of the simulation plant as shown in table 4, simulation results show that this method can effectively use $3 \mathrm{D}$ Terrestrial LiDAR data to describe the production of given plants which is consistent with the reality, and has good practicality.

\begin{tabular}{|c|c|c|c|c|c|}
\hline $\begin{array}{c}\text { Target } \\
\text { trees }\end{array}$ & $\begin{array}{c}\text { Branch } \\
\text { number }\end{array}$ & $\begin{array}{c}\text { Layer } \\
\text { number }\end{array}$ & $\begin{array}{c}\text { Total } \\
\text { number } \\
\text { of } \\
\text { nodes }\end{array}$ & $\begin{array}{c}\text { Number } \\
\text { of } \\
\text { growth } \\
\text { units }\end{array}$ & $\begin{array}{c}\text { Number of } \\
\text { productions }\end{array}$ \\
\hline Oak & $\begin{array}{c}105 \\
(127) \\
35\end{array}$ & 8 & 232 & 1376 & 12 \\
Locust & $(63)$ & 7 & 91 & 2365 & 8 \\
\hline
\end{tabular}

Table 4. Experimental data

\section{CONCLUSION}

On the base of the study on the characteristics of Terrestrial LiDAR data as well as $3 \mathrm{D}$ L-system, we present a 3D reconstruction method of branching structures of given plants based on the Terrestrial LiDAR data combined with L-system. Simulation results show that this method effectively obtained branching structures which consistent with the real ones of given plants and realized 3D reconstruction of their branches. Using algorithms to extract the topology structures of plants and then extract the characteristics of production, the previous Lsystem has been improved, while it relied on the method of a large number of experimental observation and analysis of statistics in the extraction of plant production which was timeconsuming, complex and uncertain.

Terrestrial LiDAR technology has been repeatedly applied in the extraction of geometrical and biological parameters of individual plant, but due to the complexity of the spatial structures of plants and the incompleteness of point cloud data, most results were not accurate and ideal enough. This article used Terrestrial LiDAR system with high spatial resolution, using close and multi-station way to obtain the complete point cloud information of the target plants, and thus remedying the shortcomings above, achieved a good data base. On the basis of complete point cloud data of the target plants, the space-level segmentation mechanism presented in this paper can better extract the spatial topology information and branch parameters of the target plants, achieving an automatic extraction of topology structures of plants by using computer algorithm.

Following the steps in this paper, through establishing algorithm to analyze the plant topology and then extracting the production, even the persons who is not very familiar with the L-system can also use the method to extract the production of plants and generate 3D models with high degree of simulation, improving the efficiency of the extraction of plant production and providing a basis for the study of the physiological and ecological processes related to plant structures, and also providing a new way for the description of plant production.

However, due to the complexity of the growing structures of plants, while extracting small branches and winding branches using the method, the results were less than ideal, and there were slightly differences between the simulation results and the original structures of plants, but that does not affect the applicability of this method. Future research following this article will focus on improving the method of parameters information extraction of the branching structure of plants by Terrestrial LiDAR data, to make it possible to identify more complex branches, to extract the correct plant production and to establish plant models which are consistent with reality.

In the future work it is needed to consider how to simulate the dynamic growth of a given plant by using plant leaves combined with the growing function in the scale of time, so as to make predictions and retrieval on growing processes of it.

\section{ACKNOWLEDGEMENTS}

Financial support for this study was provided through National Key Technology R\&D Program (2014BAH34B01). We are grateful to the undergraduate students and staff of the Laboratory of Forest Management and "3S" technology, Beijing Forestry University, and the great support from Beijing Municipal Bureau of Forest. A special thank is due to LI, from Tsinghua University for careful revision of English text.

\section{REFERENCES}

Bedrich Benes, Erik Uriel Millian. (2002). Virtual climbing plants competing for space. Proceedings of the Computer Animation 2002. Geneva, Switzerland. 19- 21(6), pp. 33-43.

Bian, R., Chen, P., Burrage, K., Hanan, J., Room, P., \& Belward, J., (2002). Derivation of L-system models from measurements of biological branching structures using genetic algorithms. Lecture Notes in Computer Science. 2358, pp. 514-524.

Christophe Godin, Hervé Sinoquet. (2005). Functionalstructural plant modeling. New Phytologist. 166(3), pp. 705-708.

De Reffye, P., Edelin, C., Franqon, J., Jaeger, M., \& Puech, C. (1988). Plant models faithful to botanical structure and development. Computer Graphics. 22(4), pp. 151-158.

Doron Rotem \& Varol, Y. L. (1978). Generation of Binary Trees from Ballot Sequences. Journal of the ACM (JACM). pp. $396-$ 404.

Fang, K., Jing, S., Shen, L. M., \& Chen, y. (2011). Threedimensional reconstruction of virtual plants branch structure based on quasi binary-tree. Transactions of the CSAE, 27(11), pp. 151-154.

Federl, P., \& Prusinkiewicz, P. (2004). Solving differential equations in developmental models of multicellular structures expressed using L-systems. Lecture Notes in Computer Science. 
pp. $3037,65-72$.

Felsberg, M., \& Sommer, G. (2001). Scale Adaptive Filtering Derived from the Laplace Equation. Pattern Recogniton. Lecture Notes in Computer Science. pp. 124-131.

Fujita, M., McGeer, P.C., \& Yang, J. C. Y. (2005). MultiTerminal Binary Decision Diagrams: An Efficient Data Structure for Matrix Representation. FORMAL METHODS IN SYSTEM DESIGN. pp. 149-169.

Gary D. Knott. (1997). A numbering system for binary trees. Communications of the ACM. pp. 113-115.

Garber, S. M., Monserud, R. A., \& Mguire, D. A. (2008). Crown recession patterns in three conifer species of the Northern Rocky Mountains. Forest Science. 54(6), pp. 633-646.

Godin,C., \& Caraglio, Y. (1998). A multiscale model of Plant topological structures. Journal of Theoretical Biology. 19(1), pp. $1-46$.

Godin, C., \& Sinoquet, H. (2005). Functional-structural plant modelling. New Phytol. 166(3), pp. 705-708.

Gorte, B., \& Pfeifer, N. (2004). Structuring laser-scanned trees using $3 \mathrm{~d}$ mathematical morphology. Proceedings of Commission V, Istanbul, Turkey. pp. 929-933.

Honda, H., Tomlinson, P. B., \& Fisher, J. B. (1982). Two geometrical models of branching of botanical trees. Annals of Botany. 49, pp. 1-11.

Hu, B. G., De Reffye, P., Zhao, X., Yan, H. P., \& Kang, M. Z. (2003). GreenLab: towards a new methodology of plant structural functional model structural aspect. Tsinghua University Press. pp. 21-35.

Jean-Louis Loday., \& María O. Ronco. (2002). Order Structure on the Algebra of Permutations and of Planar Binary Trees. JOURNAL OF ALGEBRAIC COMBINATORICS. pp. 253-270.

Karwowski, R., \& Prusinkiewicz, P. (2003). Design and implementation of the $\mathrm{L}+\mathrm{C}$ modeling language. Electronic Notes in Theoretical Computer Science. 86, 19.

Kniemeyer, O. (2004). Rule-based modelling with the XL/GroIMP software.In: Schaub H, Detje F, Brüggemann U, eds. The logic of Artificial Life. Proceedings of the 6th GWAL. Berlin: AKA Akademische Verlagsgesellschaft, pp. 56-65.

Lacointe, A., Isebrands, J. G., \& Host, G. E. (2002). A new way to account for the effect of source-sink spatial relationships in whole plant carbon allocation models. Canadian Journal of Forest Research. 32, pp. 1838-1848.

Lindenmayer, A. (1968). Mathematical models for cellular interaction in development, Parts I and Parts II. Journal of Theoretical Biology. 19, pp. 280-315.

Liu, Y. L., Wang, J., Chen, X., Guo, Y. W., \& Peng, Q. S. (2008) A Robust and Fast Non-Local Means Algorithm for Image Denoising. COMPUTER SCIENCE. JOURNAL OF COMPUTER SCIENCE AND TECHNOLOGY. pp. 270-279.
Magdics, M. (2009). Real-time generation of 1-system scene models for rendering and interaction. Spring conference on computer graphics. Comenius University. pp. 77-84.

McCormack, J. (2005). Aesthetic evolution of L-systems revisited. Applications of Evolutionary Computing. pp. 475-486.

Měch, R., \& Prusinkiewicz, P. (1996). Visual models of plants interacting with their environment. Proceedings of SIGGRAPH 96, New Orleans, LA, 4-9 August 1996. ACM SIGGRAPH, pp. 397-410.

Omasa, K., Hosoi, F., \& Konishi, A. (2007). 3D LiDAR imaging for detecting and understanding plant responses and canopy structure. Journal of experimental botany. 58(4), pp. 881-898.

Parker, G. G., Harding, D. J., \& Berger, M. L. (2004). A portable LiDAR system for rapid determination of forest canopy structure. Journal of Applied Ecology. 41, pp. 755-767.

Pradal, C., Dufour-Kowalski, S., Boudon, F., Fournier, C., \& Godin, C. (2008). OpenAlea: a visual programming and component-based software platform for plant modelling. Functional Plant Biology. 35, pp. 751-760.

Prusinkiewicz, P. (1998). Modeling of spatial structure and development of plants. Scientia Horticulturae. 74, pp. 113-149.

Prusinkiewicz, P. (2004). Art and science for life: designing and growing virtual plants with L-Systems. Acta Horticulturae. 630, pp. $15-28$

Prusinkiewicz, Z. (2004). Modeling plant growth and development. Current Opinion in Plant Biology. 7(1), pp. 79-83.

Prusinkiewicz, P., \& Rolland-Lagan, A. G. (2006). Modeling plant morphogenesis. Current Opinion in Plant Biology. 9, pp. 83-88.

Rakocevic, M., Sinoquet, H., \& Christophe, A. (2000). Assessing the geometric structure of a white clover canopy using 3-D digitising. Annals of Botany. 86, pp. 519-526.

Reeves, W. T. (1983). Particle systems-A technique for modeling a class of fuzzy objects. Computer Graphics. 17(3), pp. 359-376.

Shlyakhter, I., Rozenoer, M.., Dorsey, J., \& Teller, S. (2001). Reconstructing 3D tree models from instrumented photographs. Computer Graphics and Applications. 21 (3), pp. 53-61.

Sinoquet, H.., Sonohat, G., Phattaralerphong, J., \& Godin, C. (2005). Foliage randomness and light interception in 3-D digitized trees: an analysis from multiscale discretization of the canopy. Plant, Cell and Environment. 28(9), pp. 1158-1170.

Todd, K., Csillag, F., \& Atkinson, P. M. (2003). Threedimensional mapping of light transmittance and foliage distribution using LiDAR. Canadian Journal of Remote Sensing. 29(5), pp. 544-555. 